Delft University of Technology

\title{
Energy Self-Sustainability in Full-Spectrum 6G
}

Hu, Jie; Wang, Qing; Yang, Kun

DOI

10.1109/MWC.001.2000156

Publication date

2021

Document Version

Final published version

Published in

IEEE Wireless Communications

\section{Citation (APA)}

Hu, J., Wang, Q., \& Yang, K. (2021). Energy Self-Sustainability in Full-Spectrum 6G. IEEE Wireless Communications, 28(1), 104-111. [9261955]. https://doi.org/10.1109/MWC.001.2000156

\section{Important note}

To cite this publication, please use the final published version (if applicable).

Please check the document version above.

\section{Copyright}

Other than for strictly personal use, it is not permitted to download, forward or distribute the text or part of it, without the consent of the author(s) and/or copyright holder(s), unless the work is under an open content license such as Creative Commons.

\section{Takedown policy}

Please contact us and provide details if you believe this document breaches copyrights.

We will remove access to the work immediately and investigate your claim. 
Green Open Access added to TU Delft Institutional Repository

'You share, we take care!' - Taverne project

https://www.openaccess.nl/en/you-share-we-take-care

Otherwise as indicated in the copyright section: the publisher is the copyright holder of this work and the author uses the Dutch legislation to make this work public. 


\title{
Energy Self-Sustainability in Full-Spectrum 6G
}

\author{
Jie Hu, Qing Wang, and Kun Yang
}

\begin{abstract}
Full-spectrum ranging from sub- $6 \mathrm{GHz}$ to $\mathrm{THz}$ and visible light will be exploited in 6G in order to reach unprecedented key-performance-indicators. However, an extraordinary amount of energy will be consumed by network infrastructure, while functions of massively deployed Internet of Everything (IoE) devices are limited by embedded batteries. Therefore, energy self-sustainable (ESS) 6G is proposed in this article. First of all, it may achieve network-wide energy efficiency by exploiting cell-free and airborne access networks as well as by implementing intelligent reflecting surfaces (IRSs). Second, by exploiting radio-frequency/visible-light signals for on-demand wireless information and energy provision (WIEP) and for enabling passive backscatter communication, "zero-energy" IoE devices may become a reality. Furthermore, loE devices actively adapt their transceivers for better performance to a dynamic environment. Case studies of cell-free and IRS based WIEP are provided for demonstrating the advantage of our proposed architecture in terms of energy self-sustainability. This article aims to provide a first glance at primary designing principles of ESS-6G.
\end{abstract}

\section{INTRODUCTION}

With 6G going toward higher spectrum (such as Tera-Hertz or $\mathrm{THz}$ ) and thus resulting in even denser networks and smaller cells, energy consumption will become a big hurdle on the way to 6G success [1]. On the infrastructure side, a huge amount of energy will be consumed for powering numerous radio-frequency (RF) chains connected to a vast number of antennas, for extraordinary broadband signal processing, for maintaining a satisfactory coverage and for tracking mobile devices with super-narrow beams. Therefore, reducing energy consumption and jointly coordinating distributed infrastructure to achieve network-wide optimal energy-efficiency constitute the first challenge in future 6G.

On the other hand, Internet of Everything (loE), envisioned as a major 6G application, means that a vast number of small devices will be connected to networks. These devices are typically either battery-powered or batteryless. In order to extend the life-cycle of IoE devices, their energy harvesting capabilities from the ambient environment have to be activated. However, communication performance of loE devices is largely constrained by intermittent energy supplies. Therefore, designing super-low-power loE transceivers and enabling an on-demand and cost-effective manner of energy supply to these IoE devices constitute the second challenge in future 6G.

We envision 6G mobile systems have to be energy self-sustainable (ESS), both on the infrastructure side (e.g., traditional base stations) and on the device side (may it be in the form of smart phones or implanted brain computer interface devices). ESS-6G is defined in the following aspects:

- Wireless energy provision (WEP) to loE devices will be a new service class as important as communication and computing.

- Both infrastructure and loE devices will operate in a super-energy-efficient manner.

There is a consensus that visible-light ( $\mathrm{VL})$ communications will be an integral part of $6 \mathrm{G}$ in addition to the traditional medium/spectrum of RF due to VL's unique properties like massive bandwidth, information security, economical to deploy, harmless to humans, and so on. This article serves to bring these two currently separate research domains, that is, RF and VL, together to provide a full-spectrum $6 \mathrm{G}$ mobile system. A joint design that inherently considers both RF and $\mathrm{VL}$, will be the methodology in particular with energy self-sustainability,

Rather than reinventing new technologies into the already crowded 6G family, we will explore how the existing members, such as $\mathrm{THz}, \mathrm{VL}$, intelligent-reflecting-surface (IRS) and artificial-intelligence $(\mathrm{Al})$, can be invoked for achieving energy self-sustainability. This exploration needs to be conducted in a holistic manner at the design stage of ESS-6G. Fortunately, each of these candidates provides promising potential to empower energy self-sustainability. This article endeavors to shed light on potential solutions to ESS issues with the hope to spark more discussions and research on this critical aspect of 6G mobile systems.

For this purpose, this article first organizes the key 6G enabling technologies into a hierarchical architecture for ESS-6G. Guided by this architecture, mechanisms to potentially enable ESS-6G are discussed from two aspects: infrastructure and IoE devices. The infrastructure relies on both central-mobile-core and distributed-access-network with a particular focus on three new features of 6G, namely cell-free access and airborne access networks (the organization of the network) as well as IRSs (the composing materials of the network). Case studies of cell-free and IRS based wireless information and energy provision (WIEP) are presented for demonstrating the advantage of our proposed architecture in terms of energy self-sustainability. For IoE devices, discussions are given 


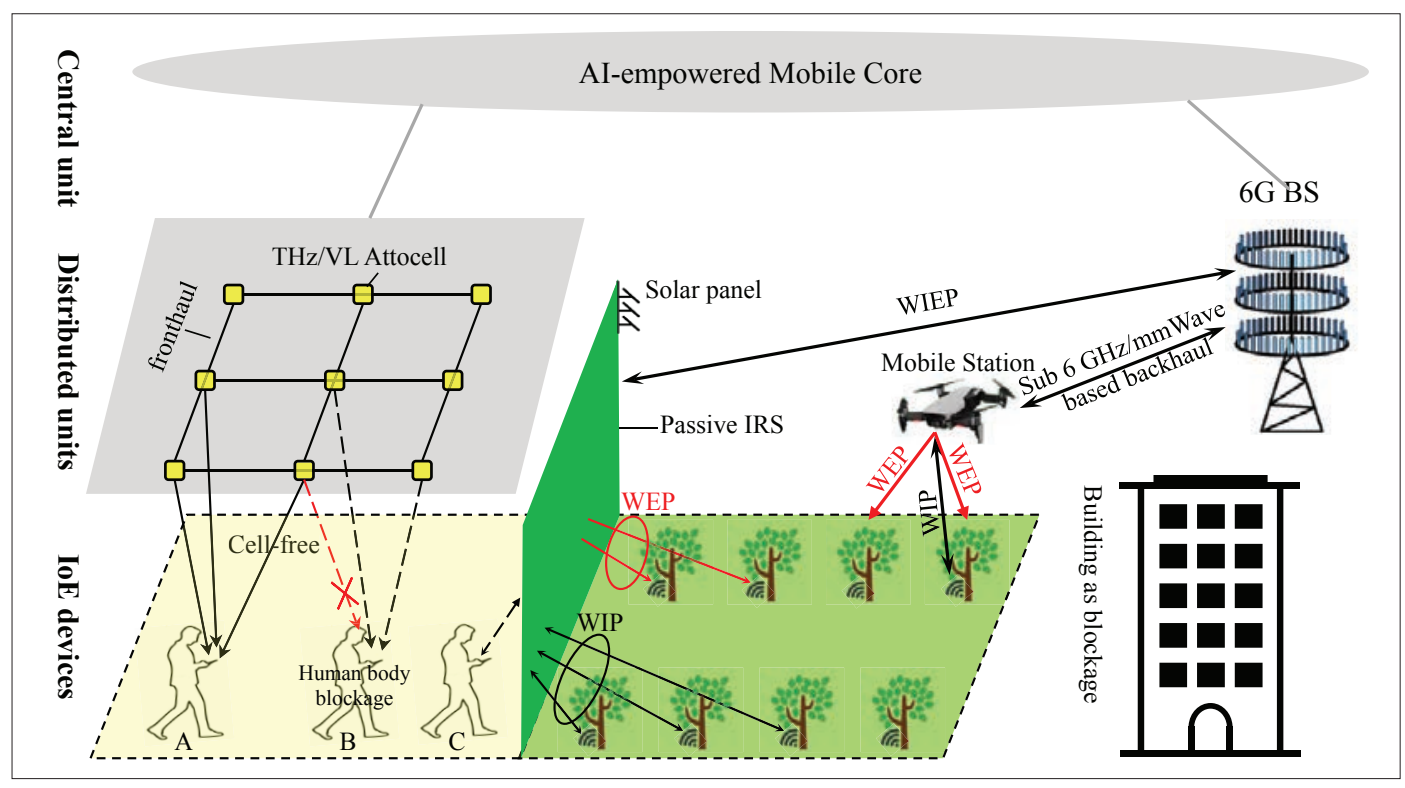

In order to achieve more stringent quality of service (QoS), $6 \mathrm{G}$

has to move up to even higher spectral

bands, such as $\mathrm{THz}$

and VL. However, 6G

may still utilize the

legacy spectrum of 5G,

such as sub- $6 \mathrm{GHz}$

and millimetre-wave

(mmWave). Therefore,

$6 \mathrm{G}$ is foreseen as a

full-spectrum mobile communication system.

to high-resolution signal processing based WIEP services, multifunctional IoE devices and human-inthe-loop based transceiver adaptation with experimental validations.

The contribution of this article is twofold. First, it proposes an architecture for ESS-6G, where traditional base stations are decomposed into two complementing layers: central units (CUs) and distributed units (DUs). CUs are analogous to today's mobile core but with more intelligence. DUs reside at very close proximity to end devices due to the employment of much higher frequency spectrum in 6G. DUs may be formed as cell-free networks, while IRSs may also be invoked as passive DUs. Second, these 6G enabling technologies are reinvestigated together from the angle of energy self-sustainability by giving insightful discussions toward a commercially viable future of ESS-6G. These investigations are centered around two complementing aspects: reducing energy consumption (i.e., energy-efficiency) and opening new WEP services. Note that introducing the details of these potential 6G enabling technologies is never the target of this article. Instead, we focus on introducing a holistic design of ESS-6G by proposing this three-layer architecture. We also focus on how we incorporate these well known techniques into different layers for achieving the ultimate energy self-sustainability.

\section{ARCHTECTURE OF ESS-6G}

In order to achieve more stringent quality of service (QoS), 6G has to move up to even higher spectral bands, such as THz and VL. However, 6G may still utilize the legacy spectrum of $5 \mathrm{G}$, such as sub-6 GHz and millimetre-wave (mmWave). Therefore, $6 \mathrm{G}$ is foreseen as a full-spectrum mobile communication system. Different spectrum will be exploited for various objectives and applications:

- Sub-6 GHz is used for large coverage and seamless handover.

- mmWave is leveraged both for wireless backhaul and fronthaul to fixed targets and for satisfying high-rate requirements of mobile devices.
- $T H z$ is invoked for providing ultra-high data rate and low-latency services for applications such as pervasive mixed-reality.

- VL with extra bandwidth is further exploited for delivering holographic tele-presence with other support of high-resolution imaging and sensing. Our proposed architecture for ESS-6G networks, as shown in Fig. 1, consists of three layers: CUs, for example, Al-empowered mobile cores; DUs, for example, self-organized attocells; and zero-energy loE devices. The Al-empowered CU determines which spectrum and which DUs are used to serve a group of IoE devices. Based on the dynamics at loE devices and surrounding environments, some DUs form exact cells to serve loE devices. Moreover, low-power multifunctional transceivers of batteryless loE devices are supported by WEP. Each layer in the architecture can contribute to the energy self-sustainability of future $6 \mathrm{G}$ networks from different dimensions.

The target of a $\mathrm{CU}$ is to provide on-demand WIEP services to IoE devices by coordinating various kinds of DUs in an energy-efficient manner and/or with green energy (e.g., produced by nearby solar panel), all contributing to network-wide energy self-sustainability, as illustrated in Fig. 1. For example, if an loE device is within the indoor area, the CU jointly coordinates fixed DUs to form arbitrary attocells for providing fast, ultra-reliable and energy-efficient wireless connectivities. An IRS based passive DU can be activated by the $\mathrm{CU}$ for assisting the WIEP service deliveries to massively deployed IoE devices. The well-tuned IRS based passive DUs may create artificial line-of-sight (LoS) transmission paths for delivering energy-efficient WIEP services. If an outdoor loE device requires urgent WIEP services, while it is not within the coverage of any fixed DUs, the CU can schedule a mobile DU (e.g., drones and mobile robots) to serve it.

Due to the complexity of decision making in dynamic environments, new Al techniques such as deep reinforcement learning (DRL) is a necessity for efficient network management and resource scheduling [2] at the CUs. To avoid overwhelming 


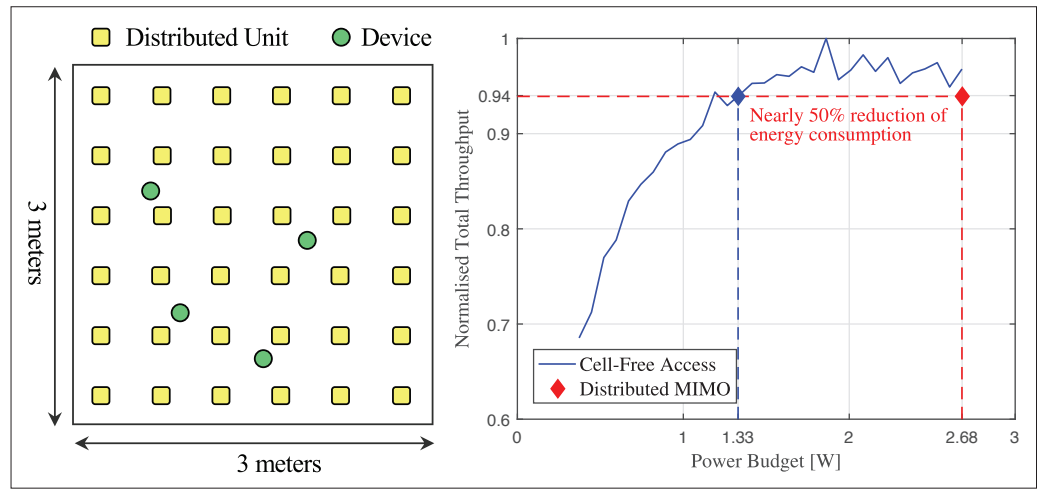

FIGURE 2. A practical experiment of cell-free access based WIP in ESS-6G.

the CUs, some intelligent processing and decision making will be conducted at DUs under the coordination of the $\mathrm{CU}$ [3]. A proper balance between the $\mathrm{CU}$ and $\mathrm{DU}$ processing is a multi-objective optimization problem that may need DRL. Software defined networking and network function virtualization will continue to evolve and they will be employed to dynamically implement new QoS algorithms or controllers on the fly.

\section{DISTRIBUTED UNITS FOR SMART ACCESS}

Toward ESS-6G, every Joule of energy should be efficiently consumed in access networks for WIEP by adopting the following methods:

- Breaking cellular boundaries for globally scheduling DUs to deliver seamless services and to achieve network-wide energy-efficiency.

- Deploying flexible airborne access network to increase the energy-efficiency of downlink/ uplink WIEP in the vertical dimension.

- Actively changing adverse wireless environment to be beneficial by implementing IRSs so as to substantially increase the efficiency of WIEP.

\section{CELL-FrEE ACCESS}

In a cell-free massive multiple-input-multiple-output (MIMO) system, a large number of DUs are connected to a $\mathrm{CU}$ via wired/wireless fronthaul. Joint coordination among these DUs results in cell-free access networks, where IoE devices are simultaneously served by cooperative DUs. Therefore, joint coordination of a CU may achieve a network-wide energy-efficiency [4]. However, full-cooperation among DUs may result in heavy tele-traffic loads on fronthaul, since it requires the exchange of network-state-information, the transmission of transmit beamformers and requested information.

In the THz/VL spectrum of 6G, signal propagation suffers from significant penetration loss, if a lot of opaque obstacles are distributed in wireless environments [5]. By further considering the increasing path-loss in high-frequency bands, super-dense DUs have to be deployed for reducing distances and for ensuring LoS between DUs and IoE devices. However, more energy has to be consumed for powering such a large-scale access network, while increased interference imposed by super-dense DUs has to be carefully managed. Furthermore, low-cost DUs can be equipped with solar panels for harvesting energy from ambient environments in order to achieve energy self-sustainability. They can be connected to smart grid to enable energy trading among their peers. As a result, global energy cooperation may result in improved network-wide energy-efficiency [6].

Due to hardware limitations, we only have limited RF chains at a $\mathrm{THz}$ based transmitter, which is far lower than the number of antennas compacted. Therefore, the accuracy of transmit beams cannot be guaranteed. An energy-efficient hybrid transmitter consisting of both digital and analog beamformers is compromised for achieving closest performance to the ideal full-digital solution [7]. Moreover, in THz/VL based cell-free access networks, the assignment of DUs and that of antennas to IoE devices can be formulated as a network-wide energy-efficiency maximization problem, while satisfying various QoS requirements of WIEP services and suppressing interference imposed by large-scale DUs and their associated antennas.

Many communication technologies can be used to achieve high-speed uplink transmissions from devices to DUs. For example, infra-red communication is very suitable for uplink transmissions, when the VL is adopted in the downlink for both illumination and communication, since it may provide high data rate and it does not have any flickering problem. This combination has been practically implemented in the state of the art, such as PureLiFi's products (https://purelifi.com/). Furthermore, in the RF band, we may also exploit broadband $\mathrm{THz}$ and IRSs for achieving high-rate and reliable uplink transmissions. The user-centric cell-free networking also provides substantial diversity gains in the space-domain for achieving high-rate and energy-efficient uplink transmissions.

We study an indoor cell-free access network with 36 DUs for the sake of validating its advantage in delivering wireless information provision (WIP) services, where the communication medium is VL. There are four devices and their positions are illustrated in the left-part of Fig. 2. We compare the normalized total throughput of the cell-free access network with that of a distributed MIMO. This normalized total throughput is defined as

Normalized Total Throughput

$$
=\frac{\text { Actual Total Throughput }}{\text { Maximum Total Throughput }} \text {, }
$$

where the maximum total throughput is obtained, when we have a power budget of $1.86 \mathrm{~W}$ in the experiment. In the cell-free access network, the DUs are dynamically assigned to the devices, which depends on the positions of the devices, the totally allowed power budget of the DUs, and the interference among the DU-device communication links. In the distributed MIMO, each device is assigned nine nearest DUs, which only depends on the positions of the devices.

According to our experimental result in the right part of Fig. 2, when the distributed MIMO achieves a normalized throughput of 0.94 , its power consumption is $2.68 \mathrm{~W}$. By contrast, the cell-free access network achieves the same normalized throughput only with a power consumption of $1.33 \mathrm{~W}$. The flexible coordination on DUs in the cell-free access network is capable of reducing nearly 50 percent power consumption for delivering the WIP services, when compared to the distributed MIMO counterpart. This experimen- 
tal result validates that organizing the DUs in the cell-free manner can efficiently reduce the energy consumption of the access network in delivering the WIP services, which is one of our main targets in ESS-6G.

\section{AIRBORNE ACCESS}

Different sorts of aircraft constitute hierarchical airborne access networks (AANs) by carrying RF/ VL transceivers on board, as illustrated in Fig. 3. Generally, based on their flying height, aircraft in AANs are classified into high-altitude platforms (HAPs) (e.g., high-altitude balloons and aircrafts) and low-altitude platforms (LAPs) (e.g., drones). AANs have to be ESS, since we cannot provide stable power supply from the ground. Joint coordination among heterogeneous aircraft is capable of extending coverage of air-to-ground communications and of realizing flexible network deployments as well as of delivering efficient WIEP services.

LAPs are mainly constituted by energy-constrained drones powered by embedded batteries, as portrayed in Fig. 3. Therefore, 3D trajectories and flight control should be carefully designed in order to reduce their energy consumption, while delivering energy-efficient downlink WEP services, downlink/uplink communication services and computation offloading services to battery-powered or batteryless ground IoE devices [8]. Moreover, the energy consumption of ground IoE devices can be substantially reduced, since flying LAPs reduce signal propagation distances to them. In addition, laser charging is an efficient way to power LAPs so as to extend their flight time, since it may focus a high amount of energy in a narrow beam. Laser beams are normally formed by ground stations or aircraft in HAPs [9].

HAPs can be aloft in the air for a relatively long time, as illustrated in Fig. 3. A key advantage of HAPs is their self-adjustable positions in order to maintain efficient WIEP services towards LAP. Moreover, HAPs can harvest stable solar energy for powering their engines and transceivers, since they are floating in the stratosphere [9].

Careful coordination among aircraft in different layers may result in a network-wide energy-efficiency and it may provide significant performance gain in the vertical dimension of ESS-6C.

\section{IRS BASED SMART ENVIRONMENT}

In all classic communication systems, only transmission or receiving strategies at transceivers can be designed for efficient WIEP [10], such as beamforming, adaptive modulation and coding design at transmitters as well as signal combining and iterative decoding design at receivers, which all aim for counteracting signal attenuation and fading in wireless channels.

As electronic materials rapidly progress, IRSs can be exploited for actively changing characteristics of wireless channels. An IRS does not have any RF chains for active transmissions. Instead, they only consist of a range of passive reflectors. Based on signals' phases and amplitudes, these programmable reflectors are intelligently adjusted, so that signals reflected by them can be constructively combined at receivers, which forms a tuned passive beam for improving the WIEP performance, as illustrated in Fig. 4a. Furthermore, observe from Fig.

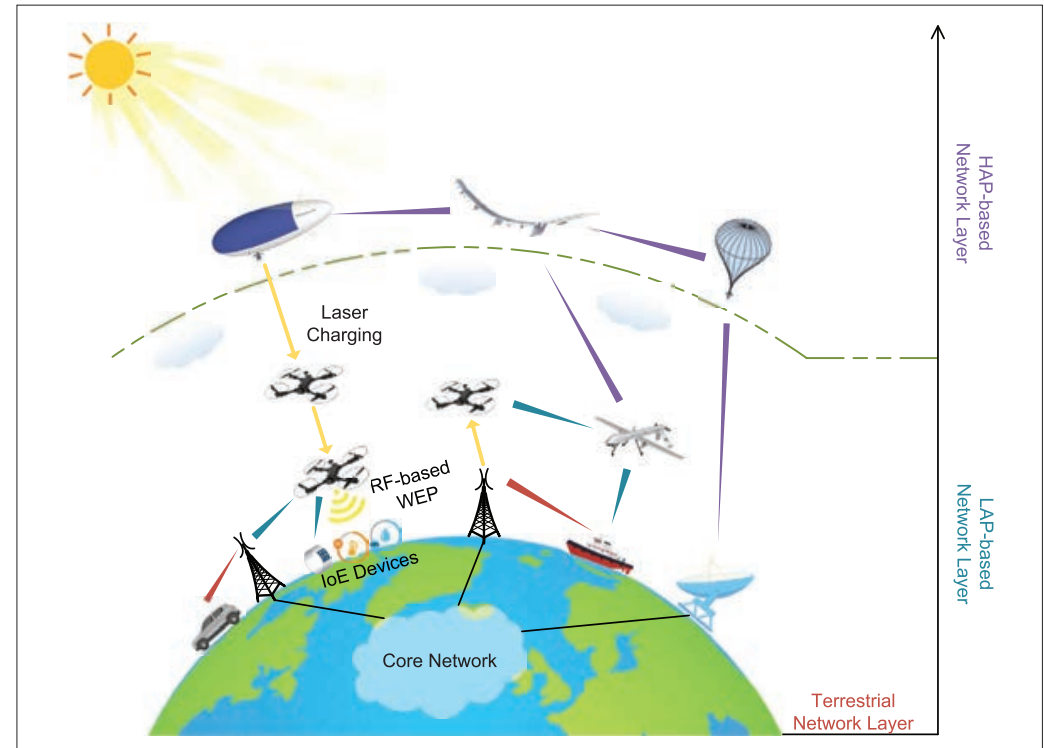

FIGURE 3. Hierarchical airborne access network.

4a that an artificial LoS link can then be established by implementing the IRS, if both the DU-to-IRS and the IRS-to-Device links are LoS, when the direct DU-to-Device link is blocked. This is essential in the $\mathrm{THz} / \mathrm{VL}$ band, since signals in these bands suffer from large penetration loss.

An IRS consumes energy for controlling its reflectors. Apart from a stable energy source, it can also be powered by RF energy harvesting for achieving its own energy self-sustainability. Signals received by a reflector are transferred to a programmable impedance-matching-network (IMN), whose reflection coefficient is adjustable. Therefore, a portion of received signals penetrate and their energy is harvested and stored. The other portion is reflected via a programmable phase-shifter. Higher reflection coefficients increase the strength of the reflected signal for achieving a better WIEP performance, while lower ones increase the energy harvested by the IRS for future usage. Moreover, carefully designed phase-shifters may result in a well-tuned beam toward the receiver. Note that adjusting programmable phase-shifters and IMNs have to consume energy harvested. Energy causality should be considered in the design of an ESS-IRS. Given insufficient energy harvested, we can only adjust several phase-shifters and IMNs, while the rest of them remain unchanged, since the energy consumption should not be higher than the energy stored in the ESS-IRS. Therefore, we have to jointly select which phase-shifters and IMNs should be adjusted and how they change the phases of reflected signals and the reflection coefficients, respectively, in order to maximize the signal strength at the receiver. By contrast, given sufficient energy harvested, more phase-shifters and more IMNs can be adjusted, which results in a better-tuned passive beam and more energy harvested for supporting the next operation.

In a nutshell, given an ESS-IRS, we need to carefully design the reflection coefficients and the phase-shifters of all the reflectors by considering limited energy available. Furthermore, as exemplified in Fig. 4a, active beamforming at transmitters, passive beamforming at IRSs, and receive combin- 


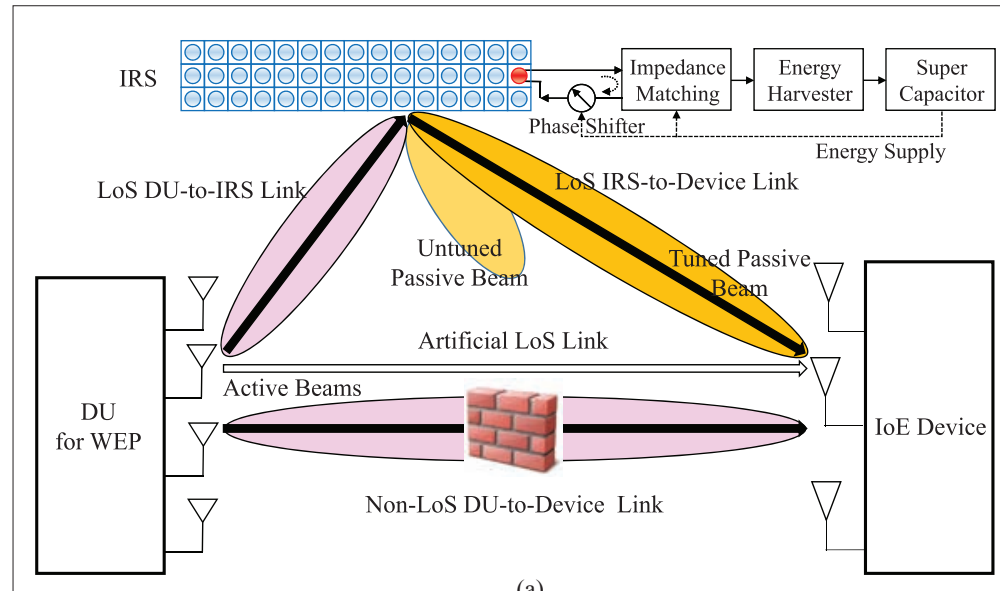

(a)

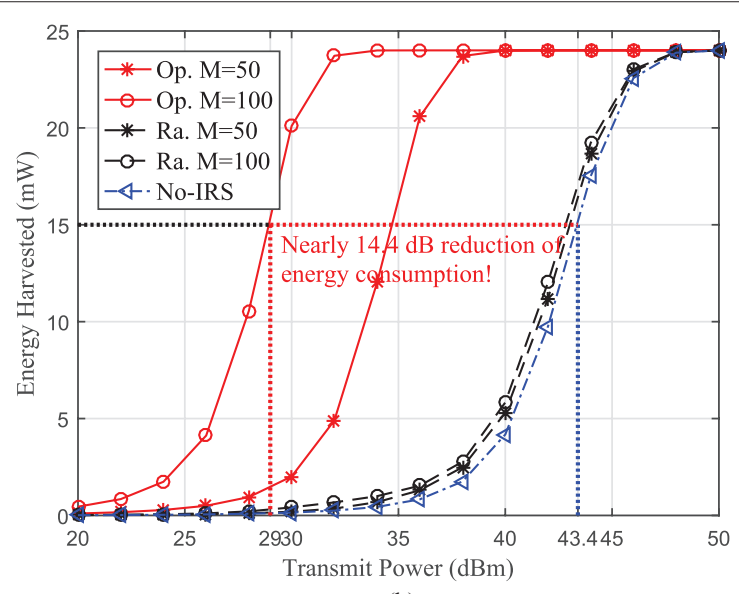

(b)

FIGURE 4. IRS based WEP in ESS-6G: a) An exemplified system model; b) Performance evaluation. The DU is equipped with 4 antennas, while the IoE device is equipped with 3 antennas. The transmit/receive antenna gains are $15 \mathrm{dBi}$ and $5 \mathrm{dBi}$. The number of reflectors in the IRS is $M=\{50,100\}$. We assume that the DU-to-IRS and IRS-to-Device links obey the Ricean fading with distances of $\{1,6\} \mathrm{m}$, respectively, and a path-loss exponent of 2, while the direct DU-to-Device link follows the Rayleigh fading with a distance of $6.5 \mathrm{~m}$ and a path-loss exponent of 4 . The reference path-loss at $1 \mathrm{~m}$ is $30 \mathrm{~dB}$. At the loE device, we consider a non-linear energy harvester with a saturation power of $24 \mathrm{~mW}$.

ing at receivers should be jointly designed to create a holographic wireless environment in ESS-6G.

Since the IRS only needs to switch the reflection coefficients of all the reflectors between one and zero [11], its energy consumption in the channel estimation is trivial. Moreover, its energy can also be effectively replenished by harvesting energy from the pilot transmissions during the channel estimation process.

We study an IRS aided WEP service from a DU to an loE device, as shown in Fig. 4a. We assume that the IRS has a stable energy source for controlling all the reflectors, while the signals can be fully reflected without any loss. In order to maximise the attainable WEP performance in terms of energy harvested, the active beamformer of the DU, the phase-shifters of the IRS, and the receive combiner of the IoE device are jointly optimized. As a benchmarker, we also consider a direct WEP service from the DU to the IoE device without the aid of the IRS as well as the random phase-shifters.

According to our simulation result in Fig. 4b, when no IRS is implemented in the wireless environment, the transmit power of the $\mathrm{DU}$ is as high as $43.4 \mathrm{dBm}$ in order to ensure $15 \mathrm{~mW}$ energy harvested by the IoE device. If an IRS having $M=$ 100 reflectors is optimally designed, for achieving the same $15 \mathrm{~mW}$ energy harvested, the DU only consumes a transmit power of $29 \mathrm{dBm}$. Actively changing the wireless environment by the IRS is capable of reducing nearly $14.4 \mathrm{~dB}$ power consumption for delivering the WEP service, when compared to the No-IRS benchmarker. Moreover, if all the phase-shifters are randomly set without any design, spatial gain provided by the IRS in the DU-to-IRS-to-Device link cannot be fully exploited. As shown in Fig. 4b, the resultant untuned passive beam only achieves a slightly higher WEP performance than the No-IRS case. The simulation result validates that the IRS based transceiver design can efficiently reduce the energy consumption of the DU in delivering the WEP services, which is another main target in ESS-6G.

\section{ZERO-ENERGY IOE DEVICES}

In order to achieve energy self-sustainability, battery-powered/batteryless IoE devices require controllable and on-demand WEP as well as low-power uplink/downlink WIP for maintaining seamless service experience. To this end, the following technologies will be adopted by loE devices:

- High-resolution signal processing based WIEP by exploiting the broadband nature of full-spectrum 6G.

- Multifunctional transceiver for batteryless communications.

- Autonomous transceiver adaptation with/without human intervention.

\section{High-Resolution Signal Processing Based WIEP}

RF signals can be exploited for flexible, controllable and on-demand WIEP at any time and anywhere. On the infrastructure side, we do not need any additional hardware implementation. Unmodulated and modulated RF signals can be relied upon for WIEP. We have to fully exploit characteristics of broadband signals for WIEP in ESS-6G, since it goes to higher spectral bands.

There are full of scatters and reflectors in the propagation of RF/VL signals, which results in multipath transmissions from a transmitter to a receiver. The number of identified paths at the receiver is determined by the following factors:

- Signal power $P_{t}$ : a higher signal power results in a higher signal-to-noise-ratio and clearer channel impulsive responses at the receiver.

- Signal bandwidth W: If the signal propagation delay between two transmission paths is higher than the coherence time $1 / W$, this pair of paths cannot be identified. Otherwise, the receiver is capable of identifying these two transmission paths.

- Geographic positions of scatters and reflectors: Given a pair of transmission paths, if their distance has a difference of $c / W$, where $c$ is the speed of light, they can be identified at the receiver, otherwise they cannot. 


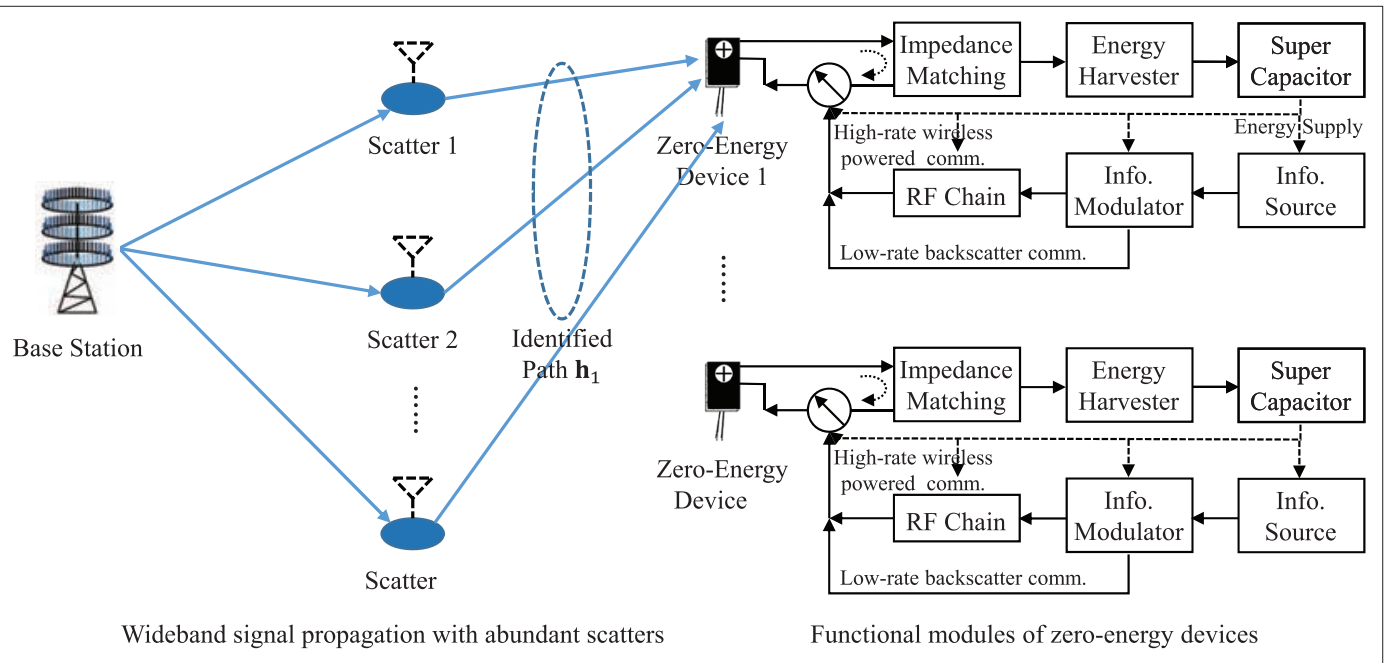

FIGURE 5. Broadband signal propagation and functional modules of zero-energy devices.
Broadband transmissions in $6 \mathrm{G}$ create a chance of high-resolution signal process-

ing on the basis of identified transmission paths, which provides substantial spatial multiplexing and diversity gains for WIEP. By fully exploiting this additional degree of freedom in signal design and the resultant performance gain, we may also

substantially reduce energy consumption of transmitters
Therefore, if we have an extremely high bandwidth $W$, more scatters and reflectors may generate more identified transmission paths [12]. We do not rely upon coarse-grained signal processing on the basis of antenna-to-antenna. Instead, broadband transmissions in 6G create a chance of high-resolution signal processing on the basis of identified transmission paths, which provides substantial spatial multiplexing and diversity gains for WIEP, as illustrated in Fig. 5. By fully exploiting this additional degree of freedom in signal design and the resultant performance gain, we may also substantially reduce energy consumption of transmitters.

\section{MUIIFUNCTIONAL TRANSCEIIER}

IoE devices should have the ability to glean energy from downlink RF/VL signals, while their energy consumption has to be suppressed. Therefore, as portrayed in Fig. 5, the following functional modules have to be implemented at IoE devices:

- An IMN, which is a circuit to guarantee that the alternate-current energy carried by the received RF/VL signals can be delivered to the back-end by matching the impedance of antennas and that of back-end's circuits.

- An energy harvester, which is a circuit for rectifying alternate-current energy carried by RF/VL signals to direct-current in order to drive electronic loads or to charge energy storage units. Key electronic elements in energy harvesters are diodes.

- An energy storage unit can be either a battery or a super-capacitor. Batteries have long-term energy storage capabilities, but their charging efficiency is low. Super-capacitors can only store energy for a short-term but they have a high charging efficiency.

Corresponding to different QoS, we have the following pair of designs for batteryless loE devices:

- High-rate wireless powered communication: IoE devices adopt a harvest-store-then-transmit protocol. In order to increase energy harvesting efficiency, we should guarantee perfect impedance matching. Only when they have sufficient energy in their storage units, their active information transmissions commence via RF/VL chains. Therefore, loE devices can obtain high- rate information transmissions [13]. However, powering RF/VL chains is an energy-consuming task.

- Low-rate backscatter communication: IoE devices deliberately mismatch the impedance of the receiving antennas and the back-end circuits. Therefore, RF/VL signals received by antennas cannot penetrate to the back-end but backscattered instead. IoE devices can flexibly modulate their own information on backscattered signals in frequency-domains and time-domains. Therefore, IoE devices do not need any RF/VL chains for information transmission, which may substantially reduce energy consumption [14]. Furthermore, we can fully exploit the abundant full-spectrum of $6 \mathrm{G}$ by intelligently changing the frequencies of backscattered signals in order to avoid congested bands. However, loE devices also need to harvest energy for powering their signal processing modules. Therefore, backscattering coefficients need to be carefully designed for allowing some of the received RF/ $\mathrm{VL}$ signals to flow into the back-end for energy harvesting. The IMN of IoE devices should also be programmable.

Furthermore, we may integrate the above-mentioned designs into a single loE device, as exemplified in Fig. 5. Therefore, low-rate control signalling or sporadic information transmissions can be completed by passive backscatter communications, while high-rate data transmissions can be completed by active wireless powered communications.

\section{HUMAN-IN-THE-LOOP}

Signal propagation in THz/VL bands of ESS-6G heavily relies on LoS between transmitters and receivers. If no LoS link exists, WIEP performance would severely degrade. In both indoor and outdoor scenarios, many objects in surrounding wireless environments may block LoS links, such as walls, cabinets, moving objects and and so on. Moreover, human bodies are mostly neglected as critical blockages in the design of a communication system operating in high-frequency bands, especially when hand-held communication devices are taken into account.

In order to improve WIEP performance, it is crucial for loE devices to possess abilities of intelligent- 
Energy consumed by the infrastructure to radiate $\mathrm{RF} / \mathrm{VL}$ signals can be significantly reduced. Based on surrounding wireless environments, loE devices may decide how to adapt themselves to reduce energy consumption in information transmission and reception as well as to increase energy harvested in downlink WEP, which is also a key step toward zero-energy devices.

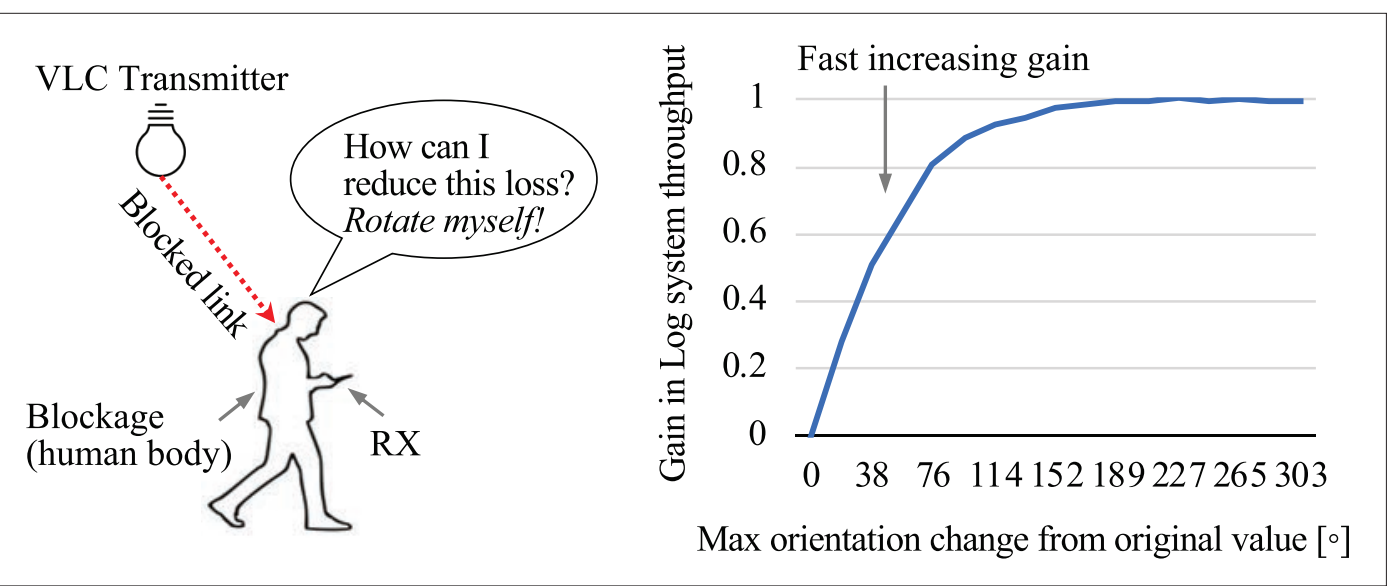

FIGURE 6. Human-in-the-loop adaptation to the surrounding environment (BX: blockage).

ly adapting their transceivers to dynamic wireless environments. This adaptation can be achieved without/with intervention from human users. For instance, reconfigurable antennas of IoE devices can be automatically adjusted to better positions in order to gain a higher information rate and to harvest more energy. Interactions between devices and human users may also result in environmental adaptation. These interactions are known as "human-in-the-loop." As illustrated in Fig. 6, human users may rotate their bodies to enable LoS links. In a case study of VL based communication networks, observe from the right part of Fig. 6 that allowing a rotation angle of $76^{\circ}$ achieves a system throughput gain of 81 percent [15]. The involvement of human users in transceiver adaptation becomes normal in our daily life. Human users holding devices always try to rotate their bodies to gain high information rates. This is more efficient in the VL band of ESS-6G, since VL can be easily captured by human eyes. Human users may readily aim their devices to intensive VL.

Autonomous transceiver adaptations naturally find wireless channels having the lowest channel attenuation. Therefore, energy consumed by the infrastructure to radiate RF/VL signals can be significantly reduced. Based on surrounding wireless environments, IoE devices may decide how to adapt themselves to reduce energy consumption in information transmission and reception as well as to increase energy harvested in downlink WEP, which is also a key step toward zero-energy devices.

\section{CONCLUSION}

This article provides a layered architecture of ESS-6G in full-spectrum which consists of Al-empowered CUs, massively deployed DUs and battery-powered or batteryless IoE devices. In order to deal with a tremendous amount of energy consumed for satisfying unprecedented QoS requirements, key solutions are provided to cell-free access for joint coordination of DUs, airborne access for 3D networking and smart wireless environmental design enabled by IRSs for counteracting severe channel attenuation in $\mathrm{THz}$ and VL. Furthermore, we provide vital paradigms to realize zero-energy loE devices, such as high-resolution signal processing based WIEP, multifunctional transceivers without any batter- ies and active transceiver adaptation to dynamic environments. However, there are still numerous challenges of making ESS-6G a reality, which calls for a joint effort from both academia and industry.

\section{ACKNOWLEDGMENT}

The authors would like to thank the financial support of the National Natural Science Foundation of China (NSFC), Grant No. 61971102, No. U1705263 and No. 61620106011. Moreover, the fiscal support of the Fundamental Research Funds for the Central Universities (Grant No. ZYGX2019J001), European Union's Horizon 2020 research and innovation program under the Marie Skodowska-Curie grant agreement, No. 824019 , are also gratefully acknowledged.

\section{REFERENCES}

[1] M. Latva-Aho and K. Leppänen, "Key Drivers and Research Challenges for 6G Ubiquitous Wireless Intelligence - 6G Research Visions 1, September 2019," Sept. 2019, pp. $1-36$.

[2] K. B. Letaief et al., "The Roadmap to 6G: Al Empowered Wireless Networks," IEEE Commun. Mag., vol. 57, no. 8, 2019, pp. 84-90.

[3] Z. Zhou et al., "Edge Intelligence: Paving the Last Mile of Artificial Intelligence with Edge Computing," Proc. IEEE, vol. 107, no. 8, 2019, pp. 1738-62.

[4] R. Jiang et al., "Joint User Association and Power Allocation for Cell-Free Visible Light Communication Networks," IEEE JSAC, 2018.

[5] F. Sheikh, Y. Gao, and T. Kaiser, "A Study of Diffuse Scattering in Massive MIMO Channels at Terahertz Frequencies," IEEE Trans. Antennas and Propagation, vol. 68, no. 2, Feb. 2020, pp. 997-1008.

[6] J. Beysens et al., "A Cell-Free Networking System with Visible Light," IEEE/ACM Trans. Networking, 2020, pp. 1-16.

[7] S. A. Busari et al., "Generalized Hybrid Beamforming for Vehicular Connectivity Using THz Massive MIMO," IEEE Trans. Vehicular Technology, vol. 68, no. 9, Sept. 2019, pp. 8372-83.

[8] Y. Du et al., "Joint Resources and Workflow Scheduling in UAV-Enabled Wirelessly-Powered MEC for loT Systems," IEEE Trans. Vehicular Technology, vol. 68, no. 10, Oct. 2019, pp. 10 187-10 200.

[9] M. Zhao, Q. Shi, and M. Zhao, "Efficiency Maximization for UAV-Enabled Mobile Relaying Systems with Laser Charging," IEEE Trans. Wireless Commun., vol. 19, no. 5, 2020, pp. 3257-72.

[10] J. Hu et al., "Integrated Data and Energy Communication Network: A Comprehensive Survey," IEEE Commun. Surveys Tutorials, vol. 20, no. 4, 4th Qtr. 2018, pp. 3169-3219.

[11] J. Chen et al., "Channel Estimation for Reconfigurable Intelligent Surface Aided Multi-User MIMO Systems," arXive, Dec. 2019; available: https://arxiv.org/abs/1912.03619

[12] Q. Xu et al., "Waveforming: An Overview with Beamforming," IEEE Commun. Surveys and Tutorials, vol. 20, no. 1, 2018, pp. 132-49. 
[13] K. Lv et al., "Throughput Maximization and Fairness Assurance in Data and Energy Integrated Communication Networks," IEEE Internet of Things J., vol. 5, no. 2, Apr. 2018, pp. 636-44.

[14] C. He et al., "Monostatic MIMO Backscatter Communications," IEEE JSAC, 2020, pp. 1-1.

[15] J. Beysens et al., "Improving Blockage Robustness in VLC Networks," Proc. Int'l. Conf. Commun. Systems Networks (COMSNETS), 2019, pp. 164-71.

\section{BIOGRAPHIES}

JIE HU [S'11, M'16] (hujie@uestc.edu.cn) received the Ph.D. degree from the School of Electronic and Computer Science, University of Southampton, U.K., in 2015. Since March 2016, he has been working with the School of Information and Communication Engineering, University of Electronic Science and Technology of China (UESTC), China, as an associate professor. His research interests include wireless communications and resource management for $\mathrm{B} 5 \mathrm{G}$ and $6 \mathrm{G}$, wireless information and energy provision and integrated communication, computing and sensing.

QING WANG [M'16] (qing@ieee.org) received the B.E. and M.S. degrees from the University of Electronic Science and Technology of China in 2008 and 2011, respectively, and the Ph.D. degree from IMDEA Networks Institute as well as the University Carlos III of Madrid in 2016. He is now an assistant professor with the Department of Software Technology, Delft University of Technology, The Netherlands. His research interests include beyond 5G networking, visible light communication, and the Internet of Things.

KUN YANG [M'00, SM'07] (kunyang@essex.ac.uk) received his Ph.D. from the Department of Electronic \& Electrical Engineering, University College London, UK. He is currently a Chair Professor in the School of Computer Science \& Electronic Engineering, University of Essex, leading the Network Convergence Laboratory, UK. His research interests include wireless networks, future Internet technology and network virtualization, mobile cloud computing and networking. 